\title{
Evaluation of whether adjuvant chemotherapy can be safely omitted for older female patients with ER-positive, HER2-negative N1 breast cancer: a study based on the SEER database
}

\author{
Minhao Lv, Peng Yuan, Youzhao Ma, Peiqi Tian, Xiuchun Chen, Zhenzhen Liu \\ Department of Breast Disease, The Affiliated Cancer Hospital of Zhengzhou University, Henan Cancer Hospital, Zhengzhou, China \\ Contributions: (I) Conception and design: X Chen, Z Liu; (II) Administrative support: Z Liu; (III) Provision of study materials or patients: All authors; \\ (IV) Collection and assembly of data: M Lv, P Yuan, Y Ma, P Tian; (V) Data analysis and interpretation: M Lv; (VI) Manuscript writing: All authors; \\ (VII) Final approval of manuscript: All authors. \\ Correspondence to: Xiuchun Chen; Zhenzhen Liu. Department of Breast Disease, The Affiliated Cancer Hospital of Zhengzhou University, Henan \\ Cancer Hospital, No. 127, Dongming Road, Zhengzhou 450008, China. Email: zlyychenxiuchun3921@zzu.edu.cn; liuzhenzhen73@126.com.
}

\begin{abstract}
Background: This study evaluated the trends and practice patterns associated with adjuvant chemotherapy (CT) use for patients aged $\geq 70$ years with estrogen receptor-positive (ER+), human epidermal growth factor receptor 2-negative (HER2-) N1 (1-3 positive lymph nodes) breast cancer (BC). Furthermore, the relationship between adjuvant CT and survival in this set of patients was determined.

Methods: The Surveillance, Epidemiology, and End Results (SEER) database was used to identify 6,711 women with ER+, HER2- N1 BC who were aged $\geq 70$ years between 2010 and 2015. Demographic, clinical, and pathological predictors of CT use were identified using logistic regression. Multivariable Cox regression was used to identify variables that correlated with overall survival (OS), before and after propensity score matching (PSM).

Results: Younger age at diagnosis, other histological types, higher tumor grade, larger tumor size, breast reconstruction surgery, progesterone receptor-negative (PR-), and increased nodal involvement were associated with an increased probability of receiving CT. CT use was associated with improved 5-year OS, both before and after PSM [hazard ratio (HR): 0.66, 95\% confidence interval (CI): 0.58-0.75 and HR: 0.81, 95\% CI: 0.68-0.96, respectively]. The exploratory subgroup analysis showed that although the benefit of CT was significant in the grade III subgroup, it was not significant in the grades I-II subgroups.

Conclusions: Adjuvant CT improved 5-year OS in patients with ER+, HER2- N1 BC who were aged $\geq 70$ years; however, the benefit of CT was more significant in the grade III subgroup than in the grades I-II subgroups.
\end{abstract}

Keywords: Breast cancer (BC); chemotherapy (CT); older women; survival; treatment de-escalation

Submitted May 28, 2021. Accepted for publication Jun 25, 2021.

doi: 10.21037/atm-21-3097

View this article at: https://dx.doi.org/10.21037/atm-21-3097

\section{Introduction}

Breast cancer (BC) is the most common malignancy among women worldwide, and its incidence increases with age; approximately $50 \%$ of $\mathrm{BC}$ cases occur in women aged $\geq 65$ years, and $>30 \%$ occur in women aged $\geq 70$ years (1). With the increase in the life expectancy of people in different populations throughout the world, there will also be an increase in the proportion of BC affecting older women.

Numerous randomized trials and meta-analyses that assessed a large number of published studies have demonstrated the efficacy of adjuvant chemotherapy (CT) for BC (2-6). Women aged $\geq 70$ years are usually excluded from most randomized clinical trials (7), so for 
the treatment of older patients with $\mathrm{BC}$, the principles associated with the treatment of their younger counterparts are generally followed.

Retrospective studies have reported a survival benefit following adjuvant $\mathrm{CT}$ in older patients with estrogen receptor-negative $(\mathrm{ER}-)$ or node-positive $\mathrm{BC}(8,9)$. However, a certain proportion of older patients have a short life expectancy and certain comorbidities, which tend to increase the toxic effects of CT and reduce treatment compliance. For older patients with ER-positive (ER+), human epidermal growth factor receptor 2-negative (HER2-) N1 (1-3 positive lymph nodes) BC, endocrine therapy (ET), which should be the mainstay of $\mathrm{BC}$ treatment, is effective and well tolerated; nevertheless, it is unclear whether older patients with ER+, HER2- N1 BC benefit from undergoing CT in addition to ET.

The aim of this study was to evaluate the trends and practice patterns of adjuvant $\mathrm{CT}$ use in patients aged $\geq 70$ years with ER+, HER2- N1 BC. Furthermore, we determined the relationship between adjuvant CT and survival in this set of patients. We present the following article in accordance with the STROBE reporting checklist (available at https://dx.doi.org/10.21037/atm-21-3097).

\section{Methods}

\section{Study population}

We retrospectively reviewed patient data from the Surveillance, Epidemiology, and End Results (SEER) database between 2010 and 2015. The SEER program, sponsored by the National Cancer Institute, comprises a consortium of 18 regional population-based cancer registries of the USA, covering approximately $30 \%$ of the population (10). All female patients aged $\geq 70$ years with ER+ and HER2- BC of stages I-III, T1-T4, and N1, whose data had been included in the SEER database, and who had received postoperative adjuvant ET, were eligible for inclusion in this study. Patients were excluded if they (I) had distant metastasis; (II) did not undergo definitive surgery; (III) had received neoadjuvant CT; (IV) had HER2positive (HER2+) or triple-negative BC; or (V) had missing data regarding treatment or follow-up. For the included patients, demographic, treatment, pathological, and survival characteristics were obtained, including year of diagnosis; age; race; histological tumor subtype; tumor grade, T stage, and stage according to the system of the American Joint
Committee on Cancer (AJCC stage, 7th edition); number of involved nodes; ER status; progesterone receptor (PR) status; HER2 status; type of surgery performed; radiationtreatment status; adjuvant-ET treatment status; adjuvantCT treatment status; and survival. The present study was based on publicly available data from the database of the SEER program, and we accessed the database with the permission number 26646-Nov2019. This study was approved by the Ethics Committee of The Affiliated Cancer Hospital of Zhengzhou University. Because all patient information in the SEER database is de-identified, informed consent was not required for this study. The study was conducted in accordance with the Declaration of Helsinki (as revised in 2013).

\section{Statistical analysis}

November 2019 was considered the follow-up cut-off point. For the estimation of overall survival (OS), the time period from the date of initial diagnosis to the date of death from any cause or the follow-up cut-off point was considered.

The demographic, clinical, and pathological characteristics were examined to predict adjuvant $\mathrm{CT}$ use in older patients with BC. Continuous variables were evaluated using the $t$-test or Wilcoxon rank-sum test. Categorical variables were evaluated using a chi-squared test or Fisher's exact test. Univariable analyses were performed to identify relevant risk factors $(\mathrm{P}<0.05)$, which were incorporated into a multivariable stepwise logistic regression model.

Based on whether CT was administered, the patients were divided into an ET group and a CT + ET group. Propensity score matching (PSM) of the ET and CT + ET groups was conducted according to age at diagnosis, year of diagnosis, race, histology, tumor grade, clinical T stage, and AJCC stage, breast surgery strategy, PR status, and regional-node positivity. PSM was conducted using a multivariable logistic regression model. The ET and CT + ET groups were matched at a ratio of 1:1 using a nearest neighbor method (11), with a caliper of 0.05 . Univariable and multivariable Cox regression analyses were used to identify variables that correlated with OS, before and after PSM. Kaplan-Meier estimator was used to calculate the 5 -year OS of each group, which were then compared using the log-rank test. All statistical analyses were performed using version 3.6.3 of the $\mathrm{R}$ software, and differences associated with $\mathrm{P}$ values $<0.05$ were considered statistically significant. 


\section{Results}

\section{Patient characteristics}

Between 2010 and 2015, a total of 6,711 female patients aged $\geq 70$ years with ER+, HER2- N1 BC were enrolled in the SEER database and included in this study. The demographic, clinical, and pathological characteristics of the patients are reported in Table 1 . Of the 6,711 patients, 1,903 (28.4\%) patients received both CT and ET, and 4,808 (71.6\%) patients received only ET. There were 2,910 (43.4\%) patients aged 70-74 years, 1,970 (29.4\%) patients aged 75-79 years, 1,199 (17.9\%) patients aged 80-84 years, and $632(9.4 \%)$ patients aged $\geq 85$ years. With respect to the clinical T stage of BC, 3,309 (49.3\%) patients had cT1, 2,860 (42.6\%) patients had cT2, 407 (6.1\%) patients had cT3, and 135 (2.0\%) patients had cT4. With respect to the grade of tumor, 1,537 (22.9\%) patients had grade I tumors, $3,739(55.7 \%)$ patients had grade II tumors, and 1,435 (21.4\%) patients had grade III tumors.

\section{Predictors of CT}

The results of adjuvant CT use in older patients are shown in Table 1. Adjuvant CT use in older patients declined dramatically with increasing age; $44.5 \%$ of those aged 70-74 years, $24.5 \%$ of those aged $75-79$ years, $8.9 \%$ of those aged $80-84$ years, and $3.0 \%$ of those $85-89$ years $(\mathrm{P}<0.001)$ received adjuvant CT. The proportion of older patients who received adjuvant CT did not change significantly over time; $28.8 \%$ of the patients diagnosed in 2010-2012 and $28.0 \%$ of the patients diagnosed in 2013-2015 received adjuvant CT $(\mathrm{P}=0.512)$.

The univariable and multivariable logistic regressions of receiving adjuvant CT by individual characteristics are shown in Table 1. Younger age at diagnosis $(\mathrm{P}<0.001)$, other histological types $(\mathrm{P}=0.021)$, higher tumor grade $(\mathrm{P}<0.001)$, larger tumor size $(\mathrm{P}<0.001)$, breast reconstruction surgery $(\mathrm{P}=0.002), \mathrm{PR}$-negative $(\mathrm{PR}-)(\mathrm{P}<0.001)$, and increased nodal involvement $(\mathrm{P}<0.001)$ were all associated with an increased probability of receiving adjuvant $\mathrm{CT}$.

\section{Survival}

The demographic and clinical characteristics of older patients with BC before and after PSM are shown in Table 2. The median follow-up, calculated using the reverse KaplanMeier estimator, was found to be 55 (interquartile range, 38-74) months (12).
The results of the univariable and multivariable Cox regression analyses performed to determine predictive factors of OS, before and after PSM, are shown in Table 3. For the entire cohort $(n=6,711)$, before PSM the Cox multivariable analysis revealed several predictors of worse OS, including older age at diagnosis, higher tumor grade, larger tumor size, $\mathrm{PR}-$, increased nodal involvement, omission of radiotherapy, and omission of CT $(\mathrm{P}<0.05$ for each predictor). For the cohort matched exactly $(n=3,122)$ after PSM, another Cox multivariable analysis also revealed several predictors of worse OS, including older age at diagnosis, higher tumor grade, larger tumor size, PR-, increased nodal involvement, omission of radiotherapy, and omission of CT $(\mathrm{P}<0.05$ for each predictor).

In the Kaplan-Meier survival curves, the OS in the CT + ET group was significantly better than that in the ET group, both before and after PSM. Before PSM, the estimated 5-year OS in the CT + ET group was $84.3 \%$ and that in the ET group was $77.4 \%$ [hazard ratio (HR): 0.66, 95\% confidence interval (CI): 0.58-0.75; $\mathrm{P}<0.001]$ (Figure 1A). After PSM, the estimated 5-year OS in the $\mathrm{CT}+\mathrm{ET}$ group was $84.2 \%$ and that in the ET group was 81.0\% (HR: 0.81; 95\% CI: 0.68-0.96; $\mathrm{P}=0.015$ ) (Figure 1B).

For the post-PSM population, we conducted an exploratory subgroup analysis and found no significant interactions between the treatment groups and subgroups. The effect of adjuvant CT on OS was consistent across all patient subgroups (Figure 2). For patients with grade I and grade II BC, the HRs for the risk of death were 1.23 (95\% CI: 0.75-1.99; P=0.41) and 0.80 (95\% CI: 0.63-1.01; $\mathrm{P}=0.06)$, respectively. For patients with grade III $\mathrm{BC}$, the HR for the risk of death was 0.69 (95\% CI: 0.51-0.93; $\mathrm{P}=0.02$ ) (Figure 2).

\section{Discussion}

For older patients with ER+, HER2- N1 BC, both undertreatment and overtreatment should be avoided. However, due to the short life expectancy of older patients and the presence of complications, it is difficult to carry out large-scale clinical trials to verify the value of adjuvant CT for this population. Although this was a retrospective study, because of the availability of data for a large number of patients aged $\geq 70$ years with ER+, HER2- N1 BC from the SEER database, we considered that it was possible and important to evaluate the effectiveness of adjuvant CT in this population using this data.

In this study, all included older patients with $\mathrm{BC}$ received 


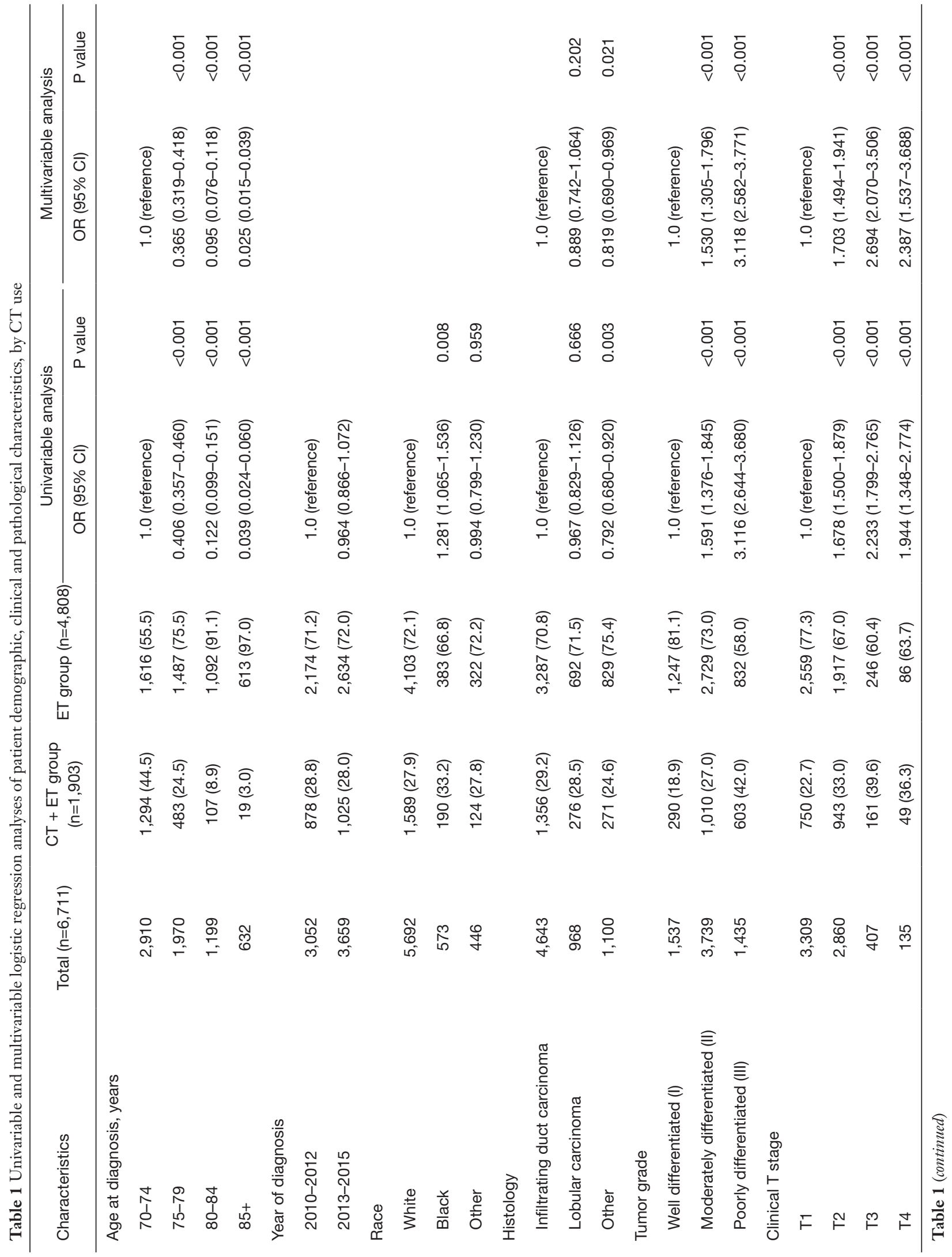




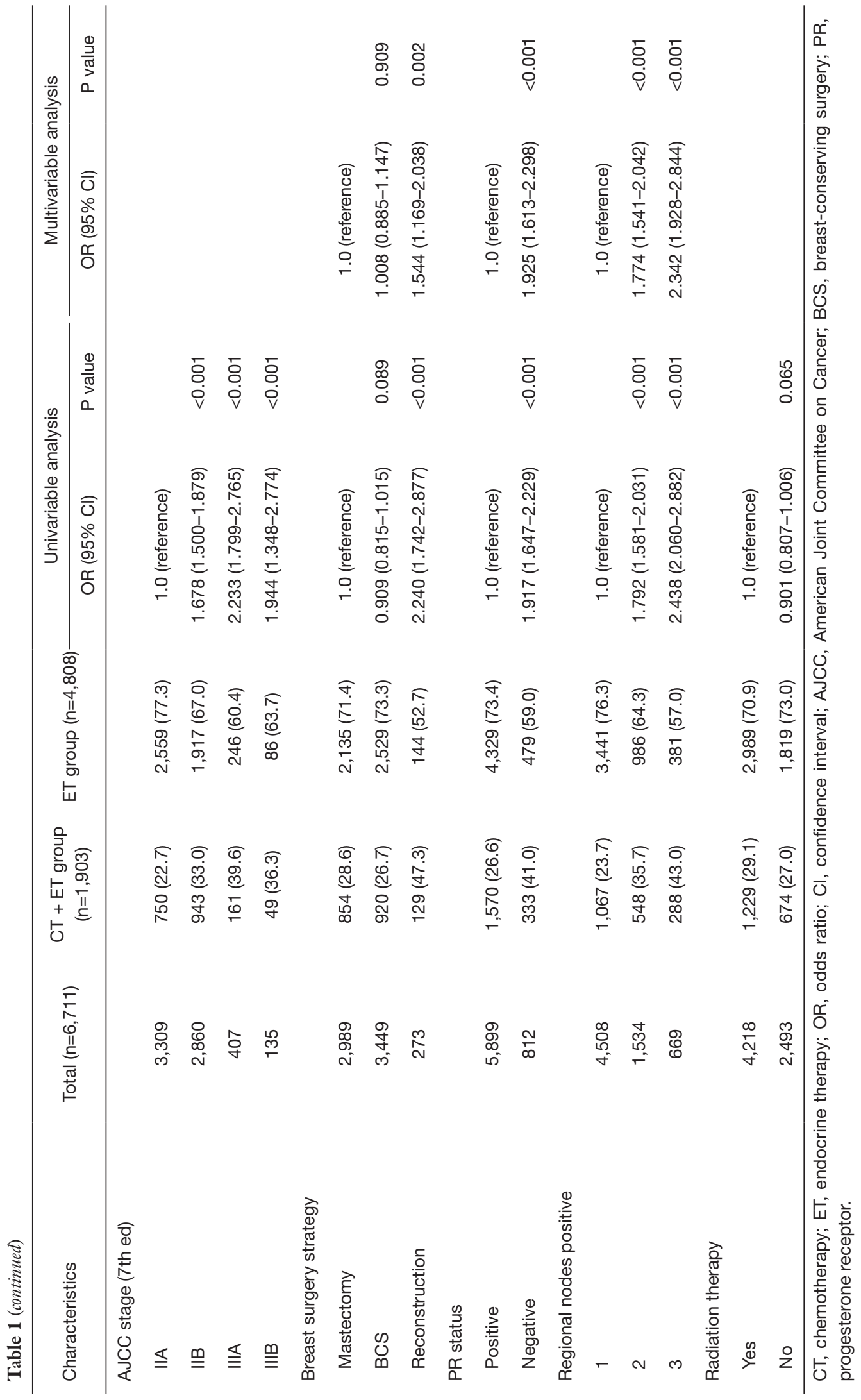




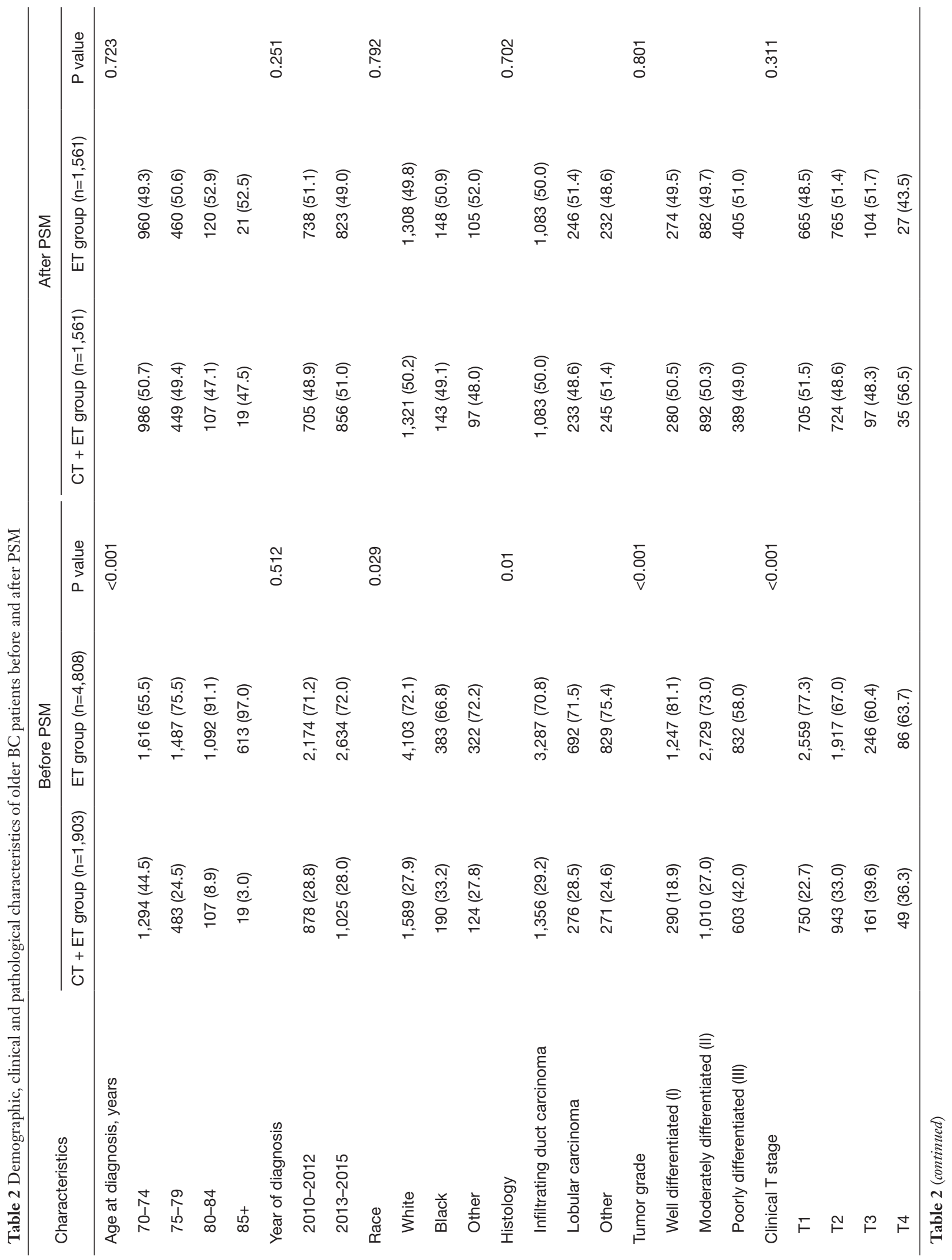




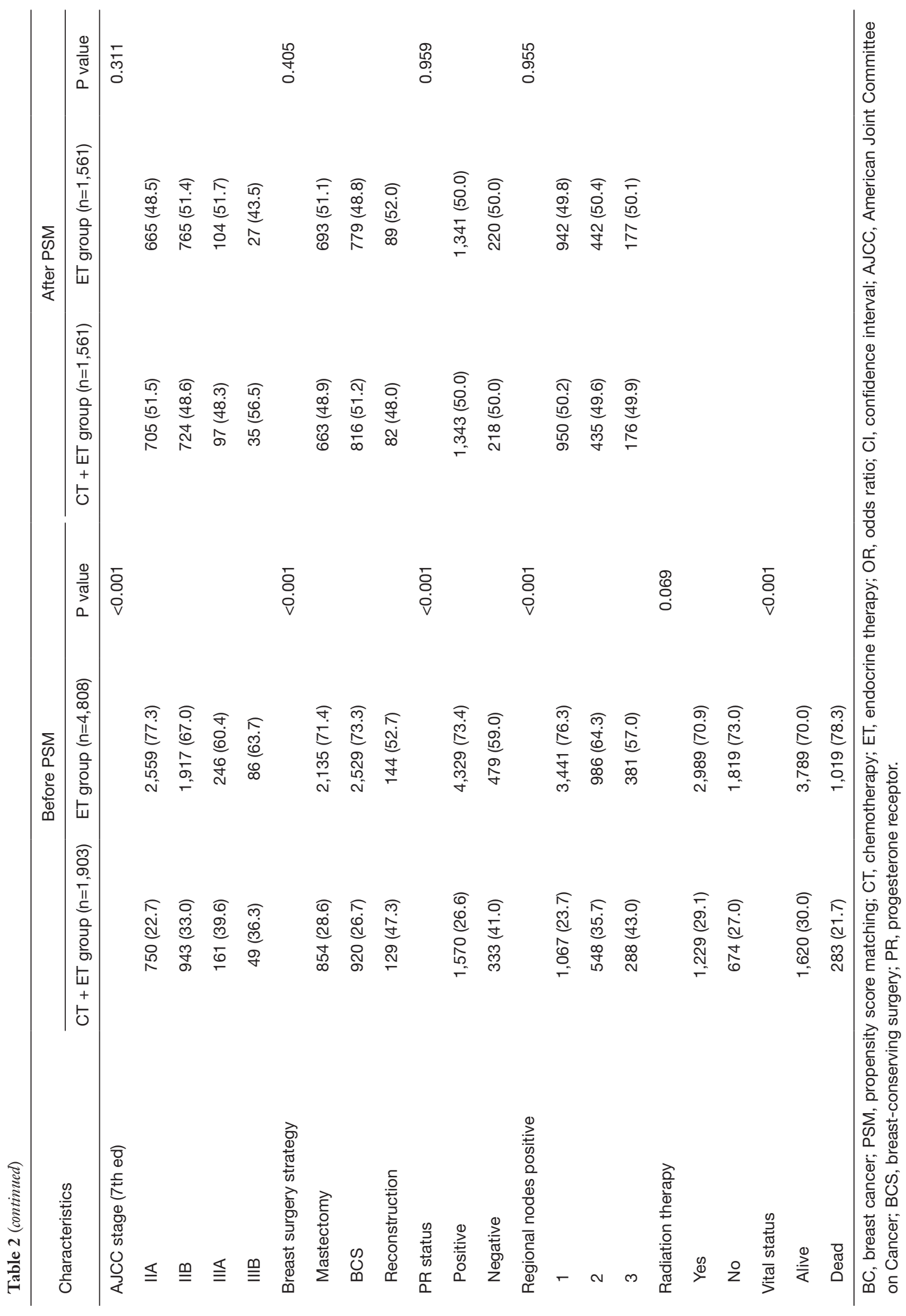




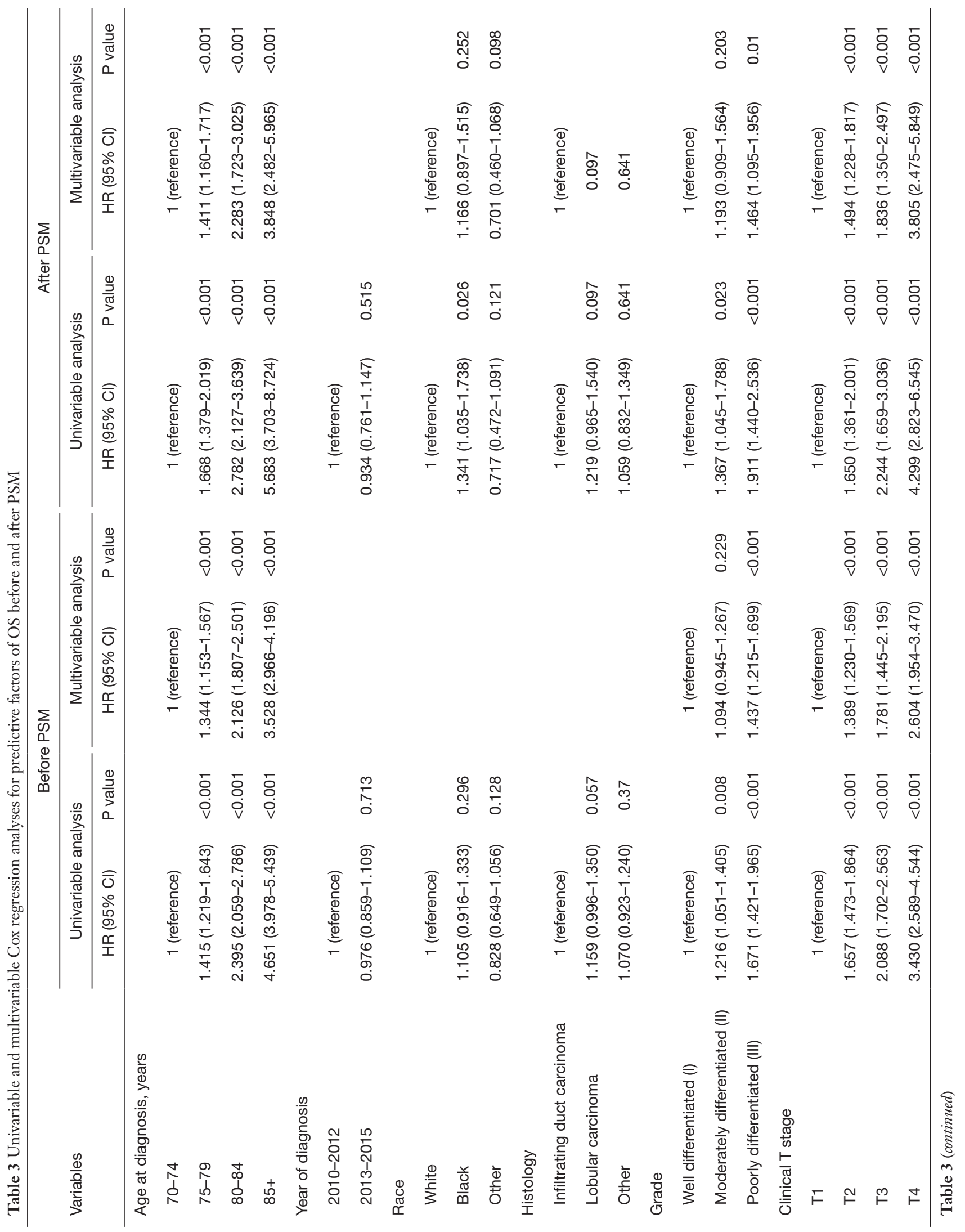




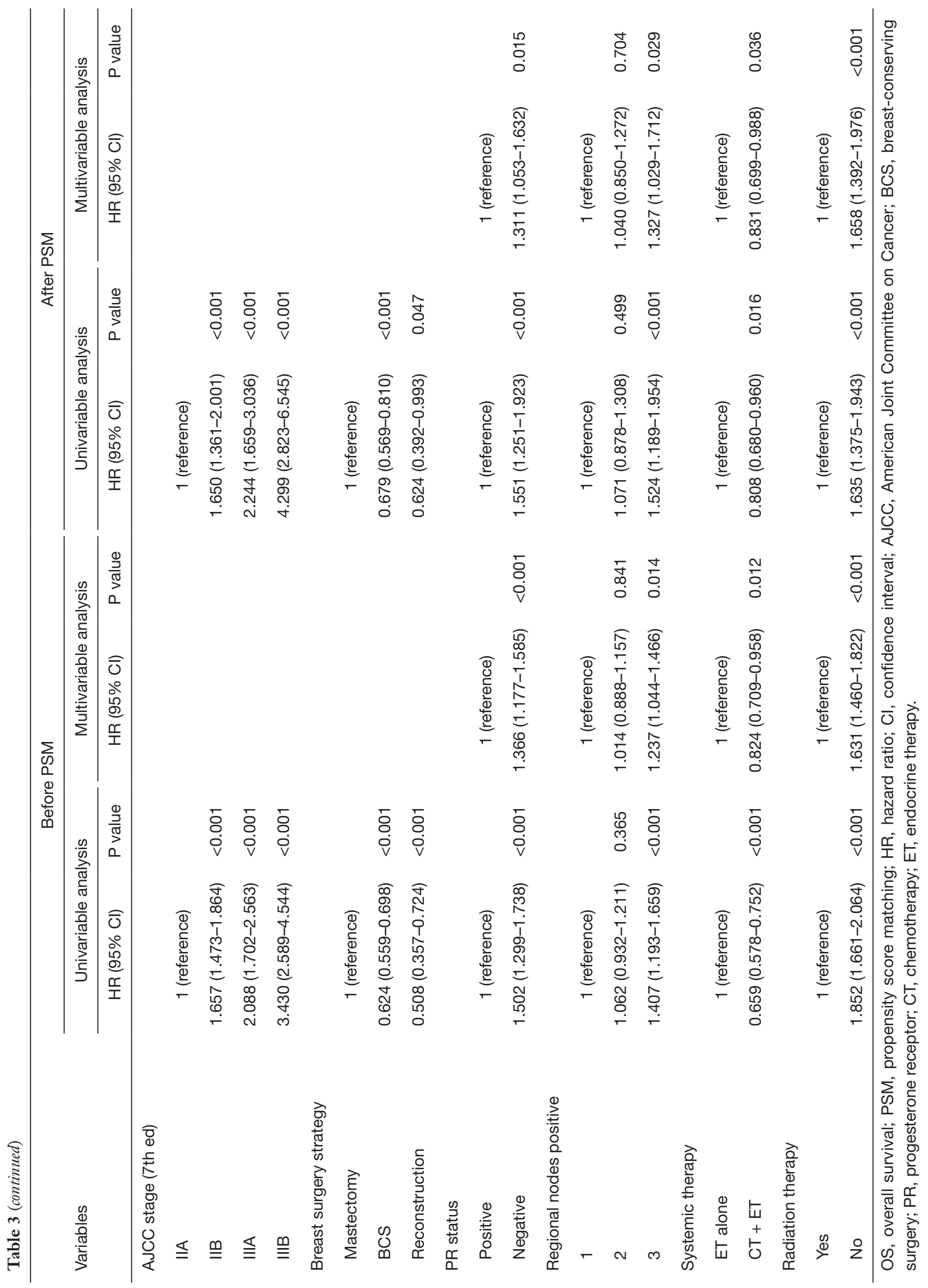



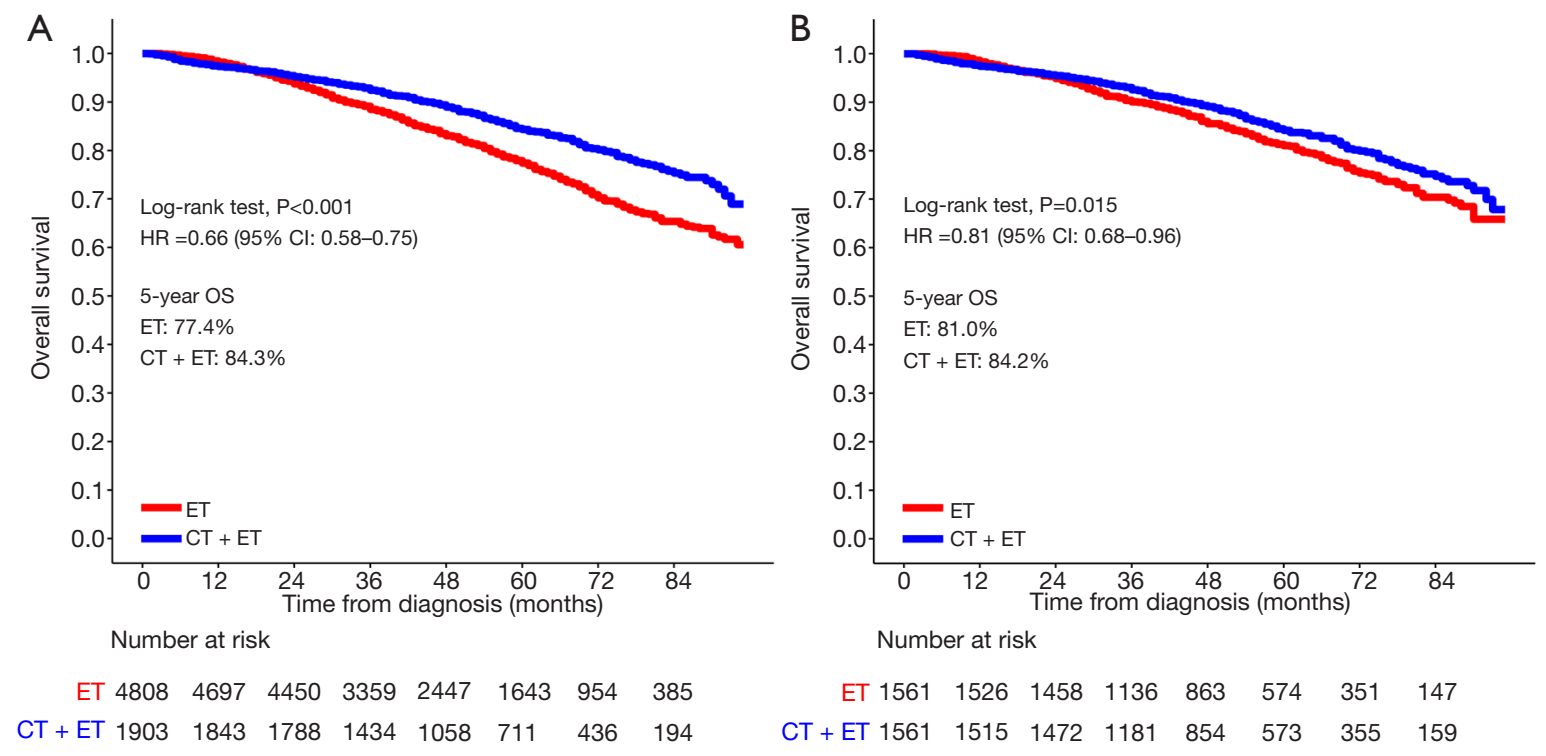

Figure 1 Kaplan-Meier curves comparing OS for the ET versus CT + ET groups in older BC patients. (A) The whole cohort (n=6,711) before PSM. (B) The exact matched cohort $(n=3,122)$ after PSM. OS, overall survival; ET, endocrine therapy; CT, chemotherapy; BC, breast cancer; PSM, propensity score matching.

adjuvant ET; however, the proportion of patients who received adjuvant CT was only $28.4 \%$, which is similar to the proportions reported in previous studies $(8,9)$. We found that although the older patients with ER+, HER2- N1 $\mathrm{BC}$ received at least systemic ET, the 5-year OS was only $79.8 \%$. This shows that although the biological behavior of $\mathrm{BC}$ in older patients is relatively good, because of certain unique aspects of the older population, some patients cannot receive standard and complete treatment. Further, most older patients have comorbidities, and consequently, with respect to the prognosis of $\mathrm{BC}$, older patients do not have any specific advantage over their younger counterparts. However, in recent years, the life expectancy of older patients with $\mathrm{BC}$ has increased significantly. It has been reported that the average remaining life expectancy is almost 16 years for a 70 -year-old woman and almost 7 years for an 85-year-old woman (13). Therefore, for older patients with ER+, HER2- N1 BC, the provision of adjuvant $\mathrm{CT}$ on the basis of age alone may negatively affect the long-term prognosis of such patients.

In this study, we found that the use of adjuvant CT decreased with increasing age among patients aged $\geq 70$ years with $\mathrm{BC}$, which is consistent with the findings of prior studies $(8,14,15)$. Among older patients, the use of adjuvant CT is limited due to a shorter life expectancy and the prevalence of comorbidities. Studies have found that before the initiation of CT for an older patient with BC, the patient's life expectancy, followed by the benefits and risks of treatment, and finally, the patient's preference must all be accounted for $(16,17)$. We should pay attention to the evidence that among older patients with $\mathrm{BC}$, the number of deaths caused by comorbidities is greater than that caused by the cancer itself $(18,19)$. Meanwhile, caution should be exercised with respect to hospitalizations for adjuvant CT-related adverse events in older patients; in one study, it was found that the hospitalization rate among women aged $>64$ years who received CT ranged from $13 \%$ to $24 \%$ (20). Other studies have found that older patients are very hesitant to receive adjuvant CT because they are more concerned about the functional decline and cognitive loss caused by CT than the survival benefits associated with CT (21). This is obviously different from the treatment preference of younger patients, whose primary concern is whether adjuvant $\mathrm{CT}$ can cause an improvement in survival. Generally speaking, treatment for older patients should be individualized, and a multidisciplinary team consultation is recommended.

The attributions of cause of deaths in death certificates are known to be error-prone and unreliable, especially for older persons $(22,23)$. Although the SEER database contains information on cause-specific death classifications abstracted from state death certificates, we did not use cancer-specific 


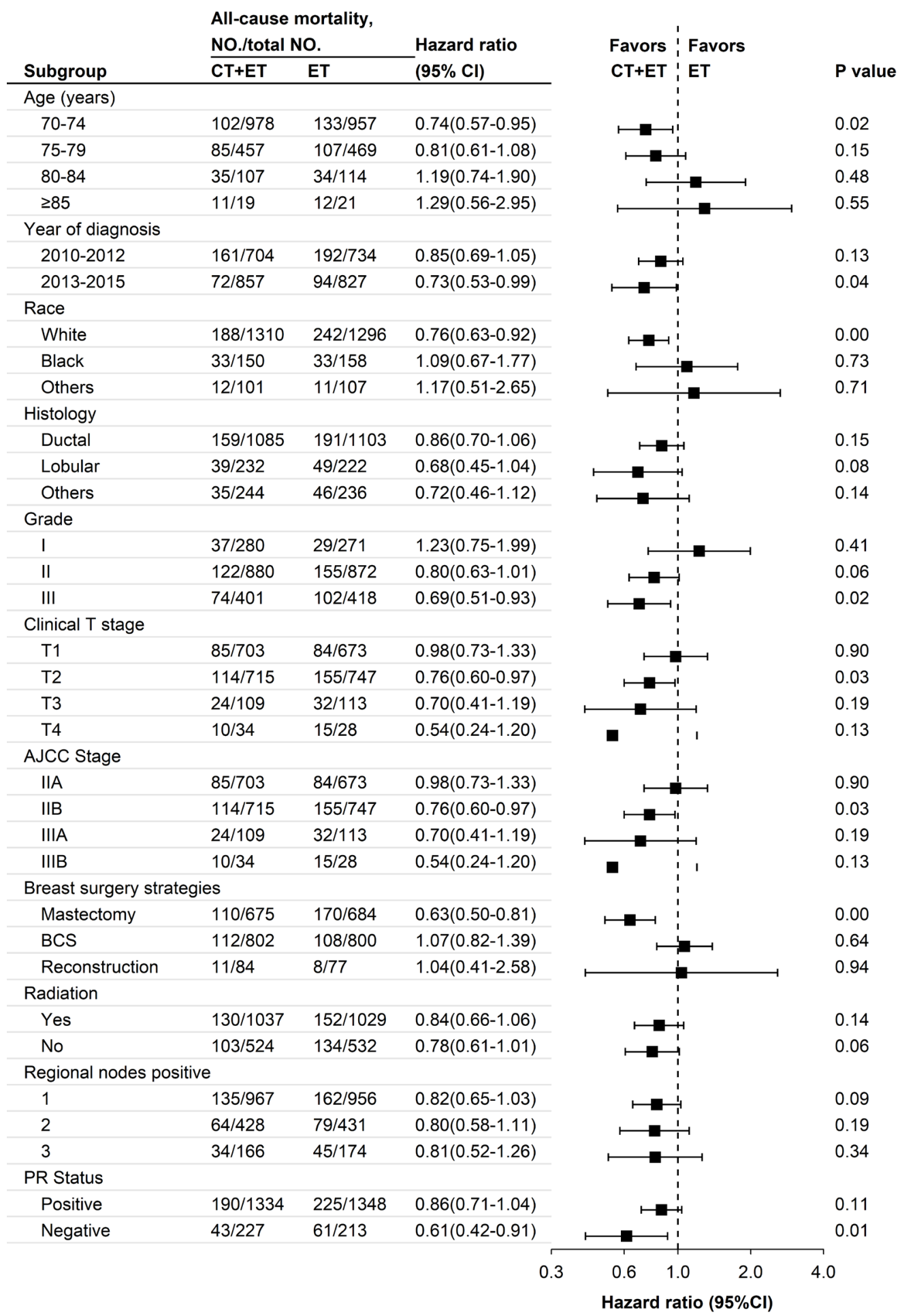

Figure 2 Subgroup analysis of all-cause death in the exact matched cohort $(n=3,122)$ after PSM. PSM, propensity score matching; CT, chemotherapy; ET, endocrine therapy; AJCC, American Joint Committee on Cancer; BCS, breast-conserving surgery; PR, progesterone receptor. 
survival as an endpoint in this study; instead, we used OS as the endpoint. In this study, both before and after PSM, Cox multivariable analyses revealed several predictors of worse OS, including older age at diagnosis, higher tumor grade, larger tumor size, PR-, increased nodal involvement, the omission of radiotherapy, and the omission of CT; these findings are similar to those reported in previous studies $(8,9)$. Meanwhile, both before and after PSM, the Kaplan-Meier survival curves and log-rank test revealed that the 5-year OS in the CT + ET group was significantly better than that in the ET group. Previous meta-analyses have suggested that adjuvant CT is associated with a $13 \%$ lower risk of all-cause death in patients with $\mathrm{BC}$ who are more than 70 years old (4). Giordano et al. also concluded that with respect to patients aged $>65$ years who had BC of clinical stages I-III, adjuvant CT was associated with a significant reduction in death from $\mathrm{BC}$ among patients with ER- and axillary lymph node-positive BC (9). It is well known that in $\mathrm{BC}$ management, efforts are continuously made to de-escalate treatment, especially for older patients with ER+, HER2- N1 BC. The prospective RxPONDER trial was designed to evaluate the benefits of $\mathrm{CT}$ in patients with ER+, HER2- N1 BC and recurrence score (RS) $\leq 25$, and its results are not expected until 2022 (24). However, in the RxPONDER trial, only $11.6 \%$ of patients were older than 70 years, and only patients with RS $\leq 25$ were enrolled. Therefore, even if the results of the RxPONDER trial are published, there will still be a lack of significant prospective research evidence that can be used to clarify the benefits of adjuvant CT in older patients with ER+, HER2- N1 BC. Our study represents one of the largest studies performed to date, in which the utilization of CT for the treatment of patients aged $\geq 70$ years with ER+, HER2- N1 BC and the effect of CT on survival were examined. Based on our findings, we believe that, at present, it may be premature and possibly inappropriate to omit adjuvant CT in this set of patients. The findings of this study may provide clinicians with accurate information that can be used when making decisions regarding the use of adjuvant CT for this population.

The exploratory subgroup analysis showed that while the benefit of adjuvant CT was significant in the subgroup of patients with grade III BC, it was not significant in the grades I-II subgroups. Lee $e t$ al. concluded that there was a significant correlation between tumor grade and RS; 95\% of patients with well-differentiated tumors had low RS, and $56 \%$ of patients with poorly/undifferentiated tumors had low RS (25). The interim analysis of the RxPONDER trial, which was presented at the 2020 San Antonio Breast Cancer Symposium, also suggested that adjuvant CT did not benefit postmenopausal patients with ER+, HER2- N1 $\mathrm{BC}$ and $\mathrm{RS} \leq 25$. Therefore, we speculate that when genetictesting tools are not available, tumor grade is an important consideration with respect to the use of adjuvant CT for older patients with ER+, HER2- N1 BC.

Several limitations of the current study should be considered. First, investigations of the SEER database are inherently retrospective; consequently, even if statistical methods such as PSM are used, problems such as selection bias cannot be eliminated completely. Second, this study used OS as the endpoint, but OS may be affected by factors unrelated to BC, such as life expectancy and comorbidities. Third, the SEER database does not provide specific information on the CT regimens used, dosages received, number of cycles of administered CT, and complications of CT. Fourth, the SEER database does not have information regarding Ki-67 percentage scores. Fifth, the SEER database does not provide accurate information regarding ET, and we considered the systemic therapy variable as ET. Sixth, the SEER database does not have information regarding duration and adherence to ET.

\section{Conclusions}

The use of adjuvant CT decreased with increasing age among patients aged $\geq 70$ years with ER+, HER2- N1 BC. The present results suggest that in this patient population, adjuvant CT improved the 5-year OS in the entire cohort; however, the subgroup analysis suggested that the benefit of CT in grade III subgroup was more significant than that in the subgroups of patients with BC of grades I-II. However, when decisions related to adjuvant $\mathrm{CT}$ are made for an older patient with BC, the patient's life expectancy and socioeconomic status, the presence of comorbidities, and the benefits and risks of CT all need to be taken into consideration.

\section{Acknowledgments}

Funding: None.

\section{Footnote}

Reporting Checklist: The authors have completed the STROBE reporting checklist. Available at https://dx.doi. org/10.21037/atm-21-3097 
Conflicts of Interest: All authors have completed the ICMJE uniform disclosure form (available at https://dx.doi. org/10.21037/atm-21-3097). The authors have no conflicts of interest to declare.

Ethical Statement: The authors are accountable for all aspects of the work in ensuring that questions related to the accuracy or integrity of any part of the work are appropriately investigated and resolved. This study was approved by the Ethics Committee of The Affiliated Cancer Hospital of Zhengzhou University. As all patient information in the SEER database is de-identified, informed consent was not necessary. This article does not contain any studies with animals performed by any of the authors. The study was conducted in accordance with the Declaration of Helsinki (as revised in 2013).

Open Access Statement: This is an Open Access article distributed in accordance with the Creative Commons Attribution-NonCommercial-NoDerivs 4.0 International License (CC BY-NC-ND 4.0), which permits the noncommercial replication and distribution of the article with the strict proviso that no changes or edits are made and the original work is properly cited (including links to both the formal publication through the relevant DOI and the license). See: https://creativecommons.org/licenses/by-nc-nd/4.0/.

\section{References}

1. Gennari R, Curigliano G, Rotmensz N, et al. Breast carcinoma in elderly women: features of disease presentation, choice of local and systemic treatments compared with younger postmenopasual patients. Cancer 2004;101:1302-10.

2. Mansour EG, Gray R, Shatila AH, et al. Efficacy of adjuvant chemotherapy in high-risk node-negative breast cancer. An intergroup study. N Engl J Med 1989;320:485-90.

3. Mansour EG, Gray R, Shatila AH, et al. Survival advantage of adjuvant chemotherapy in high-risk nodenegative breast cancer: ten-year analysis--an intergroup study. J Clin Oncol 1998;16:3486-92.

4. Early Breast Cancer Trialists' Collaborative Group (EBCTCG). Effects of chemotherapy and hormonal therapy for early breast cancer on recurrence and 15year survival: an overview of the randomised trials. Lancet 2005;365:1687-717.

5. Lv M, Li J, Guo H, et al. Impact of ipsilateral supraclavicular lymph node dissection (ISLND) for breast cancer patients and a nomogram for predicting ipsilateral supraclavicular pathological complete response (ispCR). Ann Surg Oncol 2021. [Epub ahead of print]. doi: 10.1245/ s10434-020-09548-6. Erratum in: Ann Surg Oncol 2021.

6. Lv M, Guo H, Wang C, et al. Neoadjuvant docetaxel with or without carboplatin plus dual HER2 blockade for HER2-positive breast cancer: a retrospective multi-center Chinese study. Gland Surg 2020;9:2079-90.

7. Hutchins LF, Unger JM, Crowley JJ, et al. Underrepresentation of patients 65 years of age or older in cancer-treatment trials. N Engl J Med 1999;341:2061-7.

8. Elkin EB, Hurria A, Mitra N, et al. Adjuvant chemotherapy and survival in older women with hormone receptor-negative breast cancer: assessing outcome in a population-based, observational cohort. J Clin Oncol 2006;24:2757-64.

9. Giordano SH, Duan Z, Kuo YF, et al. Use and outcomes of adjuvant chemotherapy in older women with breast cancer. J Clin Oncol 2006;24:2750-6.

10. Duggan MA, Anderson WF, Altekruse S, et al. The Surveillance, Epidemiology, and End Results (SEER) program and pathology: toward strengthening the critical relationship. Am J Surg Pathol 2016;40:e94-102.

11. Geldof T, Popovic D, Van Damme N, et al. Nearest neighbour propensity score matching and bootstrapping for estimating binary patient response in oncology: a monte carlo simulation. Sci Rep 2020;10:964.

12. Schemper M, Smith TL. A note on quantifying followup in studies of failure time. Control Clin Trials 1996;17:343-6.

13. Arias E. United States life tables, 2002. Natl Vital Stat Rep 2004;53:1-38.

14. Ward SE, Holmes GR, Ring A, et al. Adjuvant chemotherapy for breast cancer in older women: an analysis of retrospective English cancer registration data. Clin Oncol (R Coll Radiol) 2019;31:444-52.

15. Haque $W$, Verma $V$, Hatch $S$, et al. Omission of chemotherapy for low-grade, luminal A N1 breast cancer: patterns of care and clinical outcomes. Breast 2018;41:67-73.

16. Muss HB. Adjuvant chemotherapy in older women with breast cancer: who and what? J Clin Oncol 2014;32:1996-2000.

17. Vinh-Hung V, Nguyen NP. Older breast cancer patients: challenges facing oncologists. Transl Cancer Res 2020;9:S1-2. 
18. Hughes KS, Schnaper LA, Bellon JR, et al. Lumpectomy plus tamoxifen with or without irradiation in women age 70 years or older with early breast cancer: long-term follow-up of CALGB 9343. J Clin Oncol 2013;31:2382-7.

19. Patnaik JL, Byers T, DiGuiseppi C, et al. Cardiovascular disease competes with breast cancer as the leading cause of death for older females diagnosed with breast cancer: a retrospective cohort study. Breast Cancer Res 2011;13:R64.

20. Barcenas CH, Niu J, Zhang N, et al. Risk of hospitalization according to chemotherapy regimen in early-stage breast cancer. J Clin Oncol 2014;32:2010-7.

21. Fried TR, Bradley EH, Towle VR, et al. Understanding the treatment preferences of seriously ill patients. N Engl J Med 2002;346:1061-6.

22. Lloyd-Jones DM, Martin DO, Larson MG, et al. Accuracy of death certificates for coding coronary heart disease as

Cite this article as: Lv M, Yuan P, Ma Y, Tian P, Chen X, Liu Z. Evaluation of whether adjuvant chemotherapy can be safely omitted for older female patients with ER-positive, HER2-negative N1 breast cancer: a study based on the SEER database. Ann Transl Med 2021;9(13):1082. doi: 10.21037/atm21-3097 the cause of death. Ann Intern Med 1998;129:1020-6.

23. Lu TH, Shih TP, Lee MC, et al. Diversity in death certification: a case vignette approach. J Clin Epidemiol 2001;54:1086-93.

24. Tamoxifen citrate, letrozole, anastrozole, or exemestane with or without chemotherapy in treating patients with invasive RxPONDER breast cancer. ClinicalTrials.gov. Available online: https:/clinicaltrials.gov/ct2/show/ NCT01272037

25. Lee RM, Switchenko JM, Ho TB, et al. Is routine recurrence score testing in patients older than 70 years of age warranted? An evaluation of the National Cancer Database after TAILORx. Ann Surg Oncol 2019;26:3152-8.

(English Language Editor: K. Brown) 